\title{
A POLYNOMIAL APPROACH TO FAST ALGORITHMS FOR DISCRETE FOURIER-COSINE AND FOURIER-SINE TRANSFORMS
}

\author{
G. STEIDL AND M. TASCHE
}

\begin{abstract}
The discrete Fourier-cosine transform (cos-DFT), the discrete Fourier-sine transform (sin-DFT) and the discrete cosine transform (DCT) are closely related to the discrete Fourier transform (DFT) of real-valued sequences. This paper describes a general method for constructing fast algorithms for the cos-DFT, the sin-DFT and the DCT, which is based on polynomial arithmetic with Chebyshev polynomials and on the Chinese Remainder Theorem.
\end{abstract}

\section{INTRODUCTION}

In this paper, we use standard notation. By $\mathbb{N}, \mathbb{Z}, \mathbb{R}$ and $\mathbb{C}$, we denote the set of positive integers, the ring of integers, the field of reals and the field of complex numbers. For two polynomials $X, Y$ we let $X \bmod Y$ signify the remainder of $X$ divided by $Y$.

One of the most important tools in numerical analysis and digital signal processing is the fast Fourier transform (FFT), which efficiently computes the discrete Fourier transform of length $N(\operatorname{DFT}(N))$, a mapping of a sequence $\mathbf{x}=\left(x_{0}, \ldots, x_{N-1}\right) \in \mathbb{C}^{N}$ to its spectrum $\hat{\mathbf{x}}=\left(\hat{x}_{0}, \ldots, \hat{x}_{N-1}\right) \in \mathbb{C}^{N}$ defined by

$$
\hat{x}_{k}:=\sum_{j=0}^{N-1} x_{j} w_{N}^{j k}, \quad w_{N}:=\exp (-2 \pi i / N) .
$$

Using polynomial arithmetic, the formulation of many FFT-algorithms can be greatly simplified and their derivation seems more natural $[1,3,10,16]$. Further, the polynomial notation can be utilized for considerations of the computational complexity of FFT's [6, 17].

In order to introduce a polynomial representation of the DFT, we represent the input sequence $\mathbf{x} \in \mathbb{C}^{N}$ of the $\operatorname{DFT}(N)$ as the polynomial

$$
X(z):=\sum_{j=0}^{N-1} x_{j} z^{j} .
$$

Received April 3, 1989; revised December 12, 1989.

1980 Mathematics Subject Classification (1985 Revision). Primary 94A11, 42-04, 33A65.

Key words and phrases. Discrete Fourier-cosine transform, discrete Fourier-sine transform, discrete cosine transform, polynomial arithmetic. Chebyshev polynomials, computational complexity, discrete Fourier transform. 


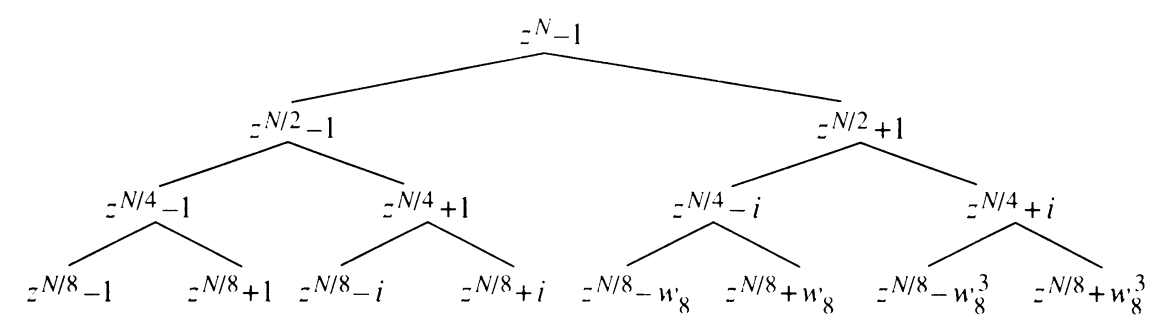

Figure 1

Factorization tree of $z^{N}-1$ with $N=2^{r} \quad(r \geq 3)$

Then we have $\hat{x}_{k}=X\left(w_{N}^{k}\right)(k=0, \ldots, N-1)$, i.e.,

$$
\hat{x}_{k}=X(z) \bmod \left(z-w_{N}^{k}\right) \quad(k=0, \ldots, N-1) .
$$

Since

$$
z^{N}-1=\prod_{k=0}^{N-1}\left(z-w_{N}^{k}\right),
$$

we get a fast algorithm for the $\operatorname{DFT}(N)$ by the Chinese Remainder Theorem (CRT) [10, pp. 26-27], if we split $X(z) \bmod \left(z^{N}-1\right)$ stepwise into equivalent simultaneous remainders, using the successive factorization of $z^{N}-1$, such that we ultimately obtain the desired simultaneous remainders (1.1). We illustrate this by the radix-2 FFT of Cooley and Tukey (see [1]).

Let $N=2^{r} \quad(r \in \mathbb{N})$. Then $z^{N}-1$ can be decomposed successively as in Figure 1. This factorization is the foundation of the radix-2 FFT, which calculates the $\operatorname{DFT}(N)$ by the recursive reduction of the input polynomial $X(z)$ modulo the factors of $z^{N}-1$ in Figure 1. The $r$ th step of this reduction procedure yields the spectrum $\hat{\mathbf{x}} \in \mathbb{C}^{N}$.

Taking into account that most DFT's are taken on real data, many fast algorithms for real DFT's were published in recent years. These algorithms exploit directly the symmetries of the real DFT [14] or use transforms, which map a real-valued sequence to a real-valued spectrum as the discrete Hartley transform [13], the DCT, the cos-DFT and the sin-DFT [15]. Although the advantage of the polynomial arithmetic for the FFT is well known, there does not exist a convenient polynomial approach to the DCT, the cos-DFT and the sin-DFT up to now. This indeed is the task of our paper. Using Chebyshev polynomials, we define the DCT, the cos-DFT and the sin-DFT on a polynomial basis. We show that this representation leads to the descriptive derivation of fast algorithms for these transforms.

Section 2, where useful properties of Chebyshev polynomials are collected, has preliminary character. In $\S 3$, we suggest a new recursive algorithm for the $\operatorname{DCT}\left(2^{r}\right)$, which works with the same number of real operations as the bestknown fast DCT's $[7,8,15]$. Introducing the cos-DFT and the sin-DFT, as well 
as their reduced versions, we apply the polynomial arithmetic to decompose the reduced cos-DFT (reduced sin-DFT) of a length $N$ divisible by 4 into a $\operatorname{DCT}(N / 4)$ and a reduced cos-DFT (reduced sin-DFT) of length $N / 2$ in $\S 5$ (cf. [15]). Our fast algorithms can be used to compute the $\operatorname{DFT}\left(2^{r}\right)$ for realand complex-valued sequences with the same computational complexity as the split-radix algorithm [3].

Although $\S \S 3$ and 5 contain mainly the special case of fast algorithms for transforms of radix-2 length, the polynomial approach to fast algorithms for the DCT, the cos-DFT, and the sin-DFT of arbitrary highly factorizable lengths will be clear. We illustrate this idea by a fast $\operatorname{DCT}(3 N)$-algorithm. Up to now, there do not exist fast algorithms for DCT's of such lengths. Note that, especially, the DCT has found wide applications in data compression and digital filtering.

\section{Chebyshev polynomials}

The polynomial approach to fast DCT's, cos-DFT's, and sin-DFT's is mainly based on known properties of Chebyshev polynomials, which we now summarize.

The Chebyshev polynomials of first and second kind can be defined recursively by

$$
\begin{aligned}
T_{0}(z) & :=1, \quad T_{1}(z):=z, \\
T_{n}(z) & :=2 z T_{n-1}(z)-T_{n-2}(z) \quad(n=2,3, \ldots), \\
T_{n} & =T_{-n} \quad(n \in \mathbb{Z}),
\end{aligned}
$$

and by

$$
\begin{aligned}
U_{0}(z) & :=1, \quad U_{1}(z):=2 z, \\
U_{n}(z) & :=2 z U_{n-1}(z)-U_{n-2}(z) \quad(n=2,3, \ldots), \\
U_{n} & =-U_{-n-2} \quad(n \in \mathbb{Z}),
\end{aligned}
$$

respectively $[11$, pp. $11-12]$. From this it follows that

$$
\begin{gathered}
T_{n}(z)=\cos (n \arccos z) \quad(|z| \leq 1 ; n \in \mathbb{Z}), \\
U_{n}(z)=\left(1-z^{2}\right)^{-1 / 2} \sin ((n+1) \arccos z) \quad(|z|<1 ; n \in \mathbb{Z}),
\end{gathered}
$$

and then

$$
\begin{array}{cc}
T_{n}(z)=2^{n-1} \prod_{k=0}^{n-1}(z-\cos (\pi(2 k+1) / 2 n)) & (n \in \mathbb{N}), \\
U_{n}(z)=2^{n} \prod_{k=1}^{n}(z-\cos (\pi k /(n+1))) & (n \in \mathbb{N}) .
\end{array}
$$

We have $[11$, p. $24 ; 12$, p. 5]

$$
T_{m n}=T_{m}\left(T_{n}\right)=2^{m-1} \prod_{k=0}^{m-1}\left(T_{n}-\cos (\pi(2 k+1) / 2 m)\right) \quad(m, n \in \mathbb{N}) .
$$


More generally, setting $y:=n \arccos z \quad(|z| \leq 1)$ in

$$
\cos m y-\cos \alpha=2^{m-1} \prod_{k=0}^{m-1}(\cos y-\cos ((\alpha+2 \pi k) / m)) \quad(m \in \mathbb{N} ; \alpha \in \mathbb{R})
$$

[5, p. 48], we obtain that

(2.6) $T_{m n}-\cos \alpha=2^{m-1} \prod_{k=0}^{m-1}\left(T_{n}-\cos ((\alpha+2 \pi k) / m)\right) \quad(m, n \in \mathbb{N} ; \alpha \in \mathbb{R})$.

Differentiation of $(2.5)$ yields

$$
\begin{aligned}
U_{m n-1} & =U_{m-1}\left(T_{n}\right) U_{n-1} \\
& =2^{m-1} \prod_{k=1}^{m-1}\left(T_{n}-\cos (\pi k / m)\right) U_{n-1} \quad(m, n \in \mathbb{N}) .
\end{aligned}
$$

Furthermore, we shall use the properties [11, p. 24]

$$
\begin{gathered}
T_{m} T_{n}=\left(T_{n-m}+T_{n+m}\right) / 2 \\
T_{m}(z) T_{n}(z)+\left(1-z^{2}\right) U_{m-1}(z) U_{n-1}(z)=T_{n-m}(z), \\
U_{n-1} T_{m}+T_{n} U_{m-1}=U_{n+m-1} .
\end{gathered}
$$

As in [4], we define the polynomials $W_{n}(n \in \mathbb{N})$ by

$$
\begin{aligned}
W_{n}(z):= & \prod_{\substack{k=1 \\
(k, n)=1}} W_{1}(z):=z-2, \quad W_{2}(z):=z+2, \\
= & \prod_{\substack{\lfloor n / 2\rfloor \\
(k, n)=1}}^{\lfloor n / 2\rfloor}(z-2 \cos (2 \pi k / n)) \quad(n=3,4, \ldots),
\end{aligned}
$$

where $\lfloor n / 2\rfloor:=\max \{k \in \mathbb{Z}: k \leq n / 2\}$ and where $(k, n)$ signifies the greatest common divisor of $k$ and $n$. For further properties of $W_{n}$, especially the connection of $W_{n}$ with Chebyshev polynomials, see [4]. Finally, let

$$
V_{m+1}(z):=\prod_{d \mid n} W_{d}(2 z)=2^{m+1} \prod_{k=0}^{m}(z-\cos (2 \pi k / n))
$$

with $m=\lfloor n / 2\rfloor$. If $n \in \mathbb{N}$ is even, then we have by (2.4) and (2.11) that

$$
V_{m+1}(z)=4\left(z^{2}-1\right) U_{m-1}(z) \text {. }
$$




\section{DiSCRETE COSINE TRANSFORM}

The discrete cosine transform of length $N(\operatorname{DCT}(N))$ is defined by the following mapping between $\mathbf{x}=\left(x_{0}, \ldots, x_{N-1}\right) \in \mathbb{R}^{N}$ and $\tilde{\mathbf{x}}=\left(\tilde{x}_{0}, \ldots, \tilde{x}_{N-1}\right) \in$ $\mathbb{R}^{N}$ :

$$
\tilde{x}_{k}:=\sum_{j=0}^{N-1} x_{j} \cos (\pi(2 k+1) j / 2 N) \quad(k=0, \ldots, N-1) .
$$

Note that our version of the $\operatorname{DCT}(N)$ is similar to the inverse DCT in $[7,9$, $15]$.

In order to introduce a polynomial notation for the DCT, we represent the $N$-point sequence $\mathbf{x} \in \mathbb{R}^{N}$ as the polynomial

$$
X:=\sum_{j=0}^{N-1} x_{j} T_{j} .
$$

Then, by $(2.1)$, we have that $(3.1)$ can be replaced by $\tilde{x}_{k}=X(\cos (\pi(2 k+1) / 2 N))$ $(k=0, \ldots, N-1)$, i.e.,

$$
\tilde{x}_{k}=X(z) \bmod (z-\cos (\pi(2 k+1) / 2 N)) \quad(k=0, \ldots, N-1) .
$$

By (2.3) and by the CRT, we obtain a fast decimation in frequency algorithm for the DCT, if we split $X \bmod T_{N}$ stepwise into the desired simultaneous remainders (3.3) by using polynomial factorizations of $T_{N}$.

In the following, let $N=2^{r} \quad(r \in \mathbb{N})$. In this case, we get a successive factorization of $T_{N}$ by applying the following

Lemma. Let $s \in \mathbb{N}(s>1)$, and let $a \in \mathbb{N}$ be an odd integer with the bit representation

$$
a=\left(a_{s-1}, \ldots, a_{1}, 1\right)_{2}:=2^{s-1} a_{s-1}+\cdots+2 a_{1}+1 \quad\left(a_{i} \in\{0,1\}\right) .
$$

By $\oplus$, we denote the addition modulo 2. Then, for any $n \in \mathbb{N}$, there holds

$$
\begin{gathered}
T_{2 n}-\cos \left(\pi a / 2^{s}\right)=2\left(T_{n}+\cos \left(\pi a / 2^{s+1}\right)\right)\left(T_{n}-\cos \left(\pi a / 2^{s+1}\right)\right), \\
T_{2 n}-\cos \left(\pi a / 2^{s}\right)=2 \prod_{j=0}^{1}\left(T_{n}-\cos \left(\pi\left(j, j \oplus a_{s-1}, \ldots, j \oplus a_{1}, 1\right)_{2} / 2^{s+1}\right)\right) .
\end{gathered}
$$

Proof. Setting $m:=2$ and $\alpha:=\pi a / 2^{s}$ in (2.6), we get (3.4). The rest of the assertion follows from

$$
\begin{aligned}
\cos \left(\pi a / 2^{s+1}\right) & =-\cos \left(\pi\left(2^{s+1}-a\right) / 2^{s+1}\right) \\
& =-\cos \left(\pi\left(2^{s}+2^{s-1}\left(1-a_{s-1}\right)+\cdots+2\left(1-a_{1}\right)+1\right) / 2^{s+1}\right) \\
& =-\cos \left(\pi\left(1,1-a_{s-1}, \ldots, 1-a_{1}, 1\right)_{2} / 2^{s+1}\right) \\
& =-\cos \left(\pi\left(1,1 \oplus a_{s-1}, \ldots, 1 \oplus a_{1}, 1\right)_{2} / 2^{s+1}\right) .
\end{aligned}
$$




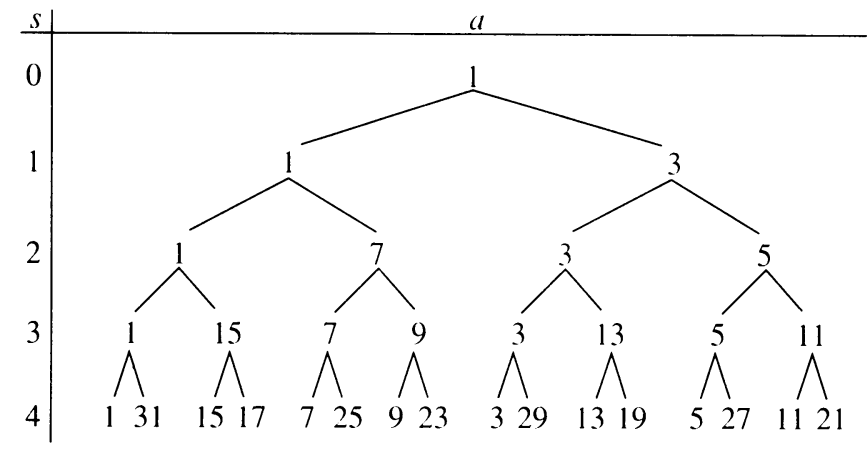

FiguRe 2

Factorization tree of $T_{N}$ with $N=2^{r} \quad(r \geq 4)$, where $a \in N$ at the sth step signifies $T_{N / 2^{\prime}}-\cos \left(\pi a / 2^{s+1}\right)$

The above lemma yields the following recursive factorization of $T_{N}=T_{N}-$ $\cos (\pi / 2)$ :

1. $T_{N}=2 T^{(0)} T^{(1)}$ with

$$
T^{\left(j_{1}\right)}:=T_{N / 2}-(-1)^{j_{1}} \sqrt{2} / 2 \quad\left(j_{1}=0,1\right) .
$$

2. $T^{\left(j_{1}\right)}=2 T^{\left(j_{1}, 0\right)} T^{\left(j_{1}, 1\right)}$ for $j_{1}=0,1$ with

$$
T^{\left(j_{1}, j_{2}\right)}:=T_{N / 4}-(-1)^{j_{2}} \cos \left(\pi\left(j_{1}, 1\right)_{2} / 8\right) \quad\left(j_{2}=0,1\right) .
$$

$r . T^{\left(j_{1}, \ldots, j_{r-1}\right)}=2 T^{\left(j_{1}, \ldots, j_{r-1} .0\right)} T^{\left(j_{1}, \ldots, j_{r-1}, 1\right)}$ for $j_{1}, \ldots, j_{r-1}=0,1$ with

$$
\begin{array}{r}
T^{\left(j_{1}, \ldots, j_{r}\right)}:=T_{1}-(-1)^{j_{r}} \cos \left(\pi\left(j_{r-1}, j_{r-1} \oplus j_{r-2}, \ldots, j_{r-1} \oplus \cdots \oplus j_{1}, 1\right)_{2} / 2 N\right) \\
\quad=T_{1}-\cos \left(\pi\left(j_{r}, j_{r} \oplus j_{r-1}, \ldots, j_{r} \oplus \cdots \oplus j_{1}, 1\right)_{2} / 2 N\right) \quad\left(j_{r}=0,1\right) .
\end{array}
$$

The $r$ th step contains the linear factors $T^{\left(j_{1}, \ldots, j_{r}\right)}$ of $T_{N}$. Figure 2 describes the decomposition of $T_{N}$ by the so-called transform factors $\cos \left(\pi\left(j_{s}, j_{s} \oplus j_{s-1}, \ldots, j_{s} \oplus \cdots \oplus j_{1}, 1\right)_{2} / 2^{s+1}\right)$.

For a given input sequence $\mathbf{x} \in \mathbb{R}^{N}$, we consider the polynomial $X$ introduced in (3.2). By the CRT, we have for every $s=1, \ldots, r$ that $X \bmod T_{N}$ is uniquely determined by its residues $X \bmod T^{\left(j_{1} \ldots . j_{1}\right)}\left(j_{1}, \ldots, j_{s}=0,1\right)$. This leads to the following recursive $\operatorname{DCT}(N)$-algorithm:

1. Calculate $X \bmod T^{\left(j_{1}\right)}$ for $j_{1}=0,1$. Observing that by (2.8)

$$
T_{N / 2+j}=2 T_{N / 2} T_{j}-T_{N / 2-j},
$$

we obtain

$$
X^{\left(j_{1}\right)}:=\sum_{j=0}^{N / 2-1} x_{j}^{\left(j_{1}\right)} T_{j}=X \bmod T^{\left(j_{1}\right)}
$$


with

$$
x_{j}^{\left(j_{1}\right)}:= \begin{cases}x_{0}+(-1)^{j_{1}} x_{N / 2} \sqrt{2} / 2 & \text { for } j=0, \\ x_{j}-x_{N-j}+(-1)^{j_{1}} x_{N / 2+j} \sqrt{2} & \text { for } j=1, \ldots, N / 2-1 .\end{cases}
$$

2. Calculate $X^{\left(j_{1}\right)} \bmod T^{\left(j_{1}, j_{2}\right)}$ for $j_{1}, j_{2}=0,1$. Using (3.5), with $N / 4$ instead of $N / 2$, we get

$$
X^{\left(j_{1}, j_{2}\right)}:=\sum_{j=0}^{N / 2-1} x_{j}^{\left(j_{1}, j_{2}\right)} T_{j}=X^{\left(j_{1}\right)} \bmod T^{\left(j_{1}, j_{2}\right)}
$$

with

$$
x_{j}^{\left(j_{1}, j_{2}\right)}:=\left\{\begin{array}{rr}
x_{0}^{\left(j_{1}\right)}+(-1)^{j_{2}} x_{N / 4}^{\left(j_{1}\right)} \cos \left(\pi\left(j_{1}, 1\right)_{2} / 8\right) & \text { for } j=0, \\
x_{j}^{\left(j_{1}\right)}-x_{N / 2-j}^{\left(j_{1}\right)}+(-1)^{j_{2}} x_{N / 4+j}^{\left(j_{1}\right)} 2 \cos \left(\pi\left(j_{1}, 1\right)_{2} / 8\right) & \text { for } j=1, \ldots, N / 4-1 .
\end{array}\right.
$$

$r$. Calculate $X^{\left(j_{1}, \ldots, j_{r-1}\right)} \bmod T^{\left(j_{1}, \ldots, j_{r}\right)}$ for $j_{1}, \ldots, j_{r}=0,1$. This yields the final result

$$
X^{\left(j_{1}, \ldots, j_{r}\right)}:=x_{0}^{\left(j_{1}, \ldots, j_{r}\right)}=X^{\left(j_{1}, \ldots, j_{r-1}\right)} \bmod T^{\left(j_{1}, \ldots, j_{r}\right)}
$$

with

$$
\begin{aligned}
x_{0}^{\left(j_{1}, \ldots, j_{r}\right)}:= & x_{0}^{\left(j_{1}, \ldots, j_{r-1}\right)}+(-1)^{j_{r}} x_{1}^{\left(j_{1}, \ldots, j_{r-1}\right)} \\
& \cdot \cos \left(\pi\left(j_{r-1}, j_{r-1} \oplus j_{r-2}, \ldots, j_{r-1} \oplus \cdots \oplus j_{1}, 1\right)_{2} / 2 N\right) .
\end{aligned}
$$

By (3.3) and by the decomposition of $T_{N}$, we see that $x_{0}^{\left(j_{1}, \ldots, j_{r}\right)}=\tilde{x}_{k}$ for the index $k$ with

$$
k=\left(j_{r}, j_{r} \oplus j_{r-1}, \ldots, j_{r} \oplus \cdots \oplus j_{1}\right)_{2}
$$

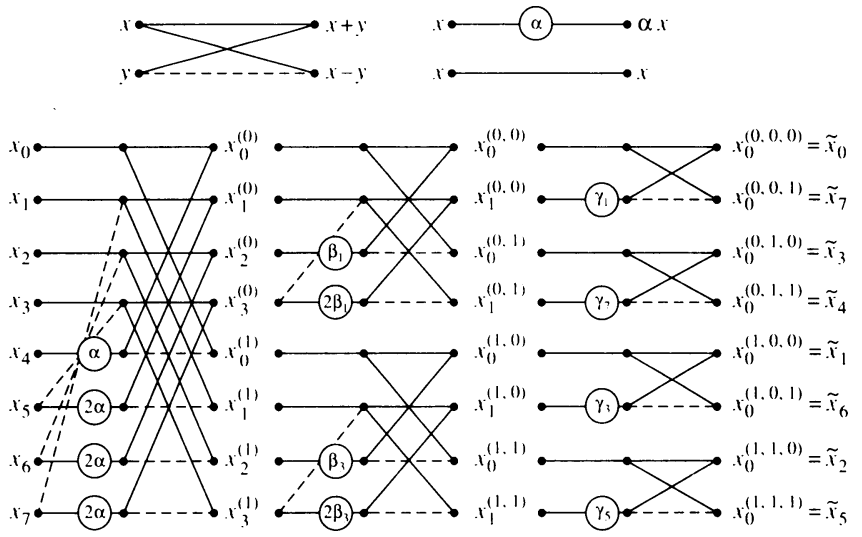

FIGURE 3

Flow graph for the $\operatorname{DCT}(8)$ with $\alpha:=\sqrt{2} / 2, \beta_{j}:=\cos (\pi j / 8)$, and $\gamma_{j}:=\cos (\pi j / 16)$ 
This describes the permutation of the output values. The $\left(j_{1}, \ldots, j_{r}\right)_{2}$ th component of our output sequence is $\tilde{x}_{k}$ with $k$ as in (3.6). Figure 3 shows the flow graph of the DCT $(8)$.

Simple considerations yield the computational complexity of our DCTalgorithm. The sth step of the algorithm requires $2^{s-1}\left(N / 2^{s}\right)$ real multiplications and $2^{s-1}\left(3 N / 2^{s}-1\right)$ real additions. Thus, our $\mathrm{DCT}(N)$-algorithm with $N=2^{r} \quad(r \in \mathbb{N})$ works with a total of

$$
\widetilde{M}_{N}=\sum_{s=1}^{r} 2^{s-1}\left(N / 2^{s}\right)=(N / 2) r
$$

real multiplications and

$$
\tilde{A}_{N}=\sum_{s=1}^{r} 2^{s-1}\left(3 N / 2^{s}-1\right)=(N / 2)(3 r-2)+1
$$

real additions. Hence, our polynomial algorithm has the same computational complexity as the best-known DCT-algorithms $[7,8,15]$.

\section{Cos-DFT AND SIN-DFT}

The image $\hat{\mathbf{x}} \in \mathbb{C}^{N}$ of the $\operatorname{DFT}(N)$ of a real-valued sequence $\mathbf{x} \in \mathbb{R}^{N}$ can be obtained by

$$
\begin{aligned}
\hat{x}_{c, k}:=\sum_{j=0}^{N-1} x_{j} \cos (2 \pi k j / N) & (k=0, \ldots, N-1), \\
\hat{x}_{s, k}:=\sum_{j=0}^{N-1} x_{j} \sin (2 \pi k j / N) & (k=0, \ldots, N-1),
\end{aligned}
$$

and by

$$
\hat{x}_{k}=\hat{x}_{c, k}-i \hat{x}_{s, k} \quad(k=0, \ldots, N-1) .
$$

The mappings defined by (4.1) and (4.2) are called the discrete Fourier-cosine transform of length $N$ (cos-DFT $(N))$ and the discrete Fourier-sine transform of length $N(\sin -\mathrm{DFT}(N))$, respectively. In this section, we present a polynomial approach to the cos-DFT and the sin-DFT, which suggests fast algorithms for both transforms.

Let $M:=\lfloor N / 2\rfloor$. By

$$
\cos (2 \pi k(N-j) / N)=\cos (2 \pi k j / N) \quad(j, k \in \mathbb{Z}),
$$

(4.1) can be rewritten as

$$
\hat{c}_{k}:=\hat{x}_{c, k}=\hat{x}_{c, N-k}=\sum_{j=0}^{M} c_{j} \cos (2 \pi k j / N) \quad(k=0, \ldots, M)
$$

with

$$
c_{j}:=\left\{\begin{array}{ll}
x_{j} & \text { for } j=0 \text { and } j=M \text { if } 2 \mid N, \\
x_{j}+x_{N-j} & \text { otherwise }
\end{array} \quad(j=0, \ldots, M) .\right.
$$


We call (4.3) the reduced cos-DFT $(N)$. Similarly, by

$$
\sin (2 \pi k(N-j) / N)=-\sin (2 \pi k j / N) \quad(j, k \in \mathbb{Z}),
$$

it follows from (4.2) that

$$
\hat{s}_{k}:=\hat{x}_{s, k}=-\hat{x}_{s, N-k}=\sum_{j=1}^{M} s_{j} \sin (2 \pi k j / N) \quad(k=1, \ldots, M)
$$

with

$$
s_{j}: c=\left\{\begin{array}{ll}
0 & \text { for } j=M \text { if } 2 \mid N, \\
x_{j}-x_{N-j} & \text { otherwise }
\end{array} \quad(j=1, \ldots, M) .\right.
$$

Then (4.4) is said to be the reduced $\sin -\operatorname{DFT}(N)$. Hence we can calculate the $\cos -\mathrm{DFT}(N) \quad(\sin -\mathrm{DFT}(N))$ by $\lceil N / 2\rceil-1$ additions and by the reduced cos$\operatorname{DFT}(N) \quad(\sin -\mathrm{DFT}(N))$. Here, $\lceil N / 2\rceil:=\min \{k \in \mathbb{Z}: k \geq N / 2\}$. In the following, we deal with these reduced transforms.

In order to introduce a polynomial notation of the reduced cos-DFT, we represent the input sequence $\mathbf{c}=\left(c_{0}, \ldots, c_{M}\right) \in \mathbb{R}^{M+1}$ as the polynomial

$$
C:=\sum_{j=0}^{M} c_{j} T_{j}
$$

Then we have by $(2.1)$ that $\hat{c}_{k}=C(\cos (2 \pi k / N)) \quad(k=0, \ldots, M)$, i.e.,

$$
\hat{c}_{k}=C(z) \bmod (z-\cos (2 \pi k / N)) \quad(k=0, \ldots, M) .
$$

By (2.11), we obtain a fast algorithm for the reduced $\cos -\operatorname{DFT}(N)$ if we split $C \bmod V_{M+1}$ stepwise into equivalent simultaneous remainders by using successive factorization of $V_{M+1}$ together with the CRT, such that we get (4.5) in the last step.

Analogously, we represent the input sequence $\mathbf{s}=\left(s_{1}, \ldots, s_{M}\right) \in \mathbb{R}^{M}$ of the reduced sin-DFT as the polynomial

$$
S:=\sum_{j=1}^{M} s_{j} U_{j-1}
$$

Then we see by (2.2) that (4.4) can be expressed as

$$
\hat{s}_{k}=\sin (2 \pi k / N) S(\cos (2 \pi k / N)) \quad(k=1, \ldots, M),
$$

i.e.,

$$
\hat{s}_{k}=\sin (2 \pi k / N) S(z) \bmod (z-\cos (2 \pi k / N)) \quad(k=1, \ldots, M) .
$$

It follows from (2.11) and from the CRT that we can deduce a fast $\sin -\mathrm{DFT}(N)$ if we reduce $S(z) \bmod \left(V_{M+1}(z) / 2(z-1)\right)$ successively into equivalent simultaneous residues by applying polynomial factorizations of $V_{M+1}(z) / 2(z-1)$, such that we ultimately obtain (4.7).

For even $N \in \mathbb{N},(4.6)$ and (4.7) can be simplified to

$$
S=\sum_{j=1}^{M-1} s_{j} U_{j-1}
$$




$$
\hat{s}_{k}=\sin (2 \pi k / N) S(z) \bmod (z-\cos (2 \pi k / N)) \quad(k=1, \ldots, M-1),
$$

since $\hat{s}_{M}=0$. By (2.4), we get a fast algorithm for the reduced $\sin -\operatorname{DFT}(N)$ with even $N \in \mathbb{N}$ by splitting $S \bmod U_{M-1}$ stepwise, such that we obtain (4.9) in the end.

\section{FAST ALGORITHMS FOR REDUCED COS-DFT AND sin-DFT}

In this section, we assume $N \in \mathbb{N}$ divisible by 4 . Set $M:=N / 2$. Based on the factorization

$$
U_{M-1}=U_{1}\left(T_{M / 2}\right) U_{M / 2-1}=2 T_{M / 2} U_{M / 2-1},
$$

which follows immediately from (2.7), we show that the reduced cos-DFT can be decomposed into the reduced cos-DFT $(N / 2)$ and the $\mathrm{DCT}(N / 4)$. The reduced sin-DFT can be handled analogously. This verifies a result in [15] from the polynomial point of view.

By (2.12), (5.1), and by the CRT, $C \bmod V_{M+1}$ is completely determined by its residues $C \bmod T_{M / 2}$ and $C \bmod V_{M / 2+1}$. First we evaluate $C \bmod T_{M / 2}$ by polynomial reductions. By $T_{j}=-T_{M-j} \bmod T_{M / 2}(j=0, \ldots, M / 2-1)$, we verify that

$$
C^{(1)}:=\sum_{j=0}^{M / 2-1} c_{j}^{(1)} T_{j}=C \bmod T_{M / 2}
$$

with $c_{j}^{(1)}:=c_{j}-c_{M-j}(j=0, \ldots, M / 2-1)$. Since by (2.12), (5.1) and (2.3),

$$
\begin{aligned}
\hat{c}_{2 k+1} & =\left(\left(C \bmod V_{M+1}\right) \bmod T_{M / 2}\right) \bmod \left(T_{1}-\cos (2 \pi(2 k+1) / N)\right) \\
& =C^{(1)} \bmod \left(T_{1}-\cos (\pi(2 k+1) / M)\right) \quad(k=0, \ldots, M / 2-1),
\end{aligned}
$$

the output values with odd indices of the reduced $\cos -\mathrm{DFT}(N)$ can be calculated by $M / 2$ additions and by the $\mathrm{DCT}(M / 2)$ given by (5.2).

On the other hand, by (2.9), we have $T_{j}=T_{M-j} \bmod V_{M / 2+1}(j=0, \ldots$, $M / 2-1)$, so that $C \bmod V_{M / 2+1}$ is obtained by

$$
C^{(2)}:=\sum_{j=0}^{M / 2} c_{j}^{(2)} T_{j}=C \bmod V_{M / 2+1}
$$

with $c_{j}^{(2)}:=c_{j}+c_{M-j}(j=0, \ldots, M / 2-1), c_{M / 2}^{(2)}:=c_{M / 2}$. Then we have by (2.12), (5.1), and (2.4) that

$$
\begin{aligned}
\hat{c}_{2 k} & =\left(\left(C \bmod V_{M+1}\right) \bmod V_{M / 2+1}\right) \bmod \left(T_{1}-\cos (2 \pi(2 k) / N)\right) \\
& =C^{(2)} \bmod \left(T_{1}-\cos (2 \pi k / M)\right) \quad(k=0, \ldots, M / 2) .
\end{aligned}
$$

Hence, we get the output values with even indices of the reduced cos-DFT $(N)$ by $M / 2$ additions and by the reduced $\cos -\mathrm{DFT}(M)$ determined in (5.3). 
Turning to the reduced sin-DFT, we take a similar approach. By (5.1) and by the CRT, $S \bmod U_{M-1}$ is completely determined by its residues $S \bmod T_{M / 2}$ and $S \bmod U_{M / 2-1}$. Considering that, by $(2.10), U_{j-1}=U_{M-j-1} \bmod T_{M / 2}$ $(j=1, \ldots, M / 2)$, we find that $S \bmod T_{M / 2}$ is given by

$$
S^{(1)}:=\sum_{j=1}^{M / 2} s_{j}^{(1)} U_{j-1}=S \bmod T_{M / 2}
$$

with $s_{j}^{(1)}:=s_{j}+s_{M-j}(j=1, \ldots, M / 2-1), s_{M / 2}^{(1)}:=s_{M / 2}$. Instead of $S^{(1)}$ we consider

$$
\tilde{S}^{(1)}:=\sum_{j=0}^{M / 2-1} \tilde{s}_{j}^{(1)} T_{j}
$$

with $\tilde{s}_{j}^{(1)}:=s_{M / 2-j}^{(1)}(j=0, \ldots, M / 2-1)$. Then from

$$
\begin{aligned}
& \sin (2 \pi(2 k+1) / N) U_{j-1}(\cos (\pi(2 k+1) / M))=\sin (2 \pi(2 k+1) j / N) \\
& \quad=(-1)^{k} \cos (2 \pi(2 k+1)(M / 2-j) / N)=(-1)^{k} T_{M / 2-j}(\cos (\pi(2 k+1) / M))
\end{aligned}
$$

one verifies that

$$
\begin{aligned}
\hat{S}_{2 k+1}= & \sin (2 \pi(2 k+1) / N)\left(\left(S \bmod U_{M-1}\right)\right. \\
& \left.\bmod T_{M / 2}\right) \\
= & \bmod \left(T_{1}-\cos (2 \pi(2 k+1) / N)\right. \\
= & (-1)^{k} \tilde{S}^{(1)} \bmod \left(T_{1}-\cos (\pi(2 k+1) / M)\right) \quad(k=0, \ldots, M / 2-1) .
\end{aligned}
$$

Consequently, we obtain the output values with odd indices of the reduced $\sin \operatorname{DFT}(N)$ by $M / 2-1$ additions and by the $\operatorname{DCT}(M / 2)$ given by (5.4), where we have to change the sign of the output values with indices congruent 3 modulo 4 .

Next, by (2.10), we have $U_{j-1}=-U_{M-j-1} \bmod U_{M / 2-1}(j=1, \ldots, M / 2-$ 1). Using this property, we form

$$
S^{(2)}:=\sum_{j=1}^{M / 2-1} s_{j}^{(2)} U_{j-1}=S \bmod U_{M / 2-1}
$$

with $s_{j}^{(2)}:=s_{j}-s_{M-j}(j=1, \ldots, M / 2-1)$. Now we conclude from

$$
\begin{aligned}
\hat{s}_{2 k} & =\sin (2 \pi(2 k) / N)\left(\left(S \bmod U_{M-1}\right) \bmod U_{M / 2-1}\right) \bmod \left(T_{1}-\cos (2 \pi(2 k) / N)\right) \\
& =\sin (2 \pi k / M) S^{(2)} \bmod \left(T_{1}-\cos (2 \pi k / M)\right) \quad(k=1, \ldots, M / 2-1),
\end{aligned}
$$

that the output values with even indices of the reduced $\sin -\operatorname{DFT}(N)$ can be evaluated by $M / 2-1$ additions and by the reduced $\sin -\mathrm{DFT}(M)$ determined in (5.5). 


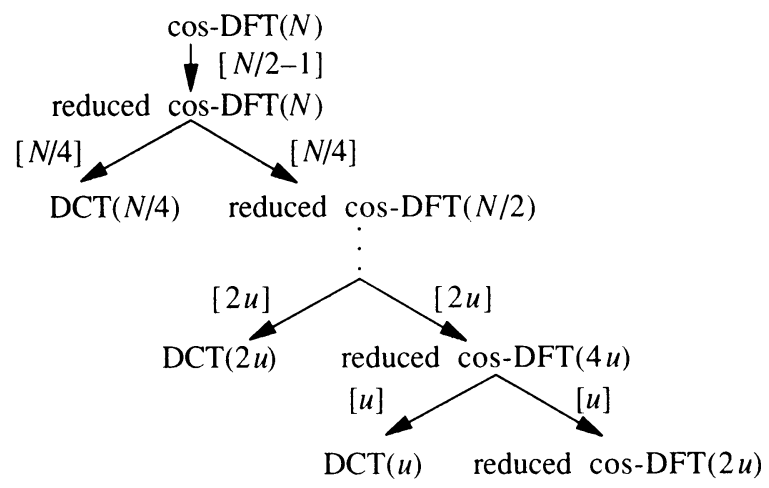

FIGURE 4

Recursive computation of the cos-DFT $(N)$ with $N=2^{r} u(r \geq$ $2 ; u$ odd $)([\cdot]$ signifies the number of additions per step $)$

Let $N=2^{r} \quad(r \geq 2)$. Then we can use the above reduction successively for the reduced cos-DFT $\left(2^{s}\right)$ (reduced $\sin -\mathrm{DFT}\left(2^{s}\right)$ ) with $s=r, \ldots, 2$. This results in the computation of the cos-DFT $(N)$ (sin-DFT $(N))$ using only DCT's and some additions. See Figure 4 with $u:=1$.

The numbers $M_{N}^{c}$ and $M_{N}^{s}$ of real multiplications and the numbers $A_{N}^{c}$ and $A_{N}^{s}$ of real additions to perform the $\cos -\operatorname{DFT}(N)$ and the $\sin -\operatorname{DFT}(N)$, respectively, follow directly from Figure 4, (3.7) and (3.8). For $N=2^{r} \quad(r \geq 2)$, one obtains

$$
\begin{aligned}
& M_{N}^{c}=M_{N}^{s}=\sum_{s=1}^{r-2} \widetilde{M}_{2^{s}}=(N / 4)(r-3)+1, \\
& A_{N}^{c}=N / 2-1+2 \sum_{s=0}^{r-2} 2^{s}+\sum_{s=1}^{r-2} \widetilde{A}_{2^{s}}+2=(N / 4)(3 r-5)+r+2, \\
& A_{N}^{s}=N / 2-1+2 \sum_{s=0}^{r-2}\left(2^{s}-1\right)+\sum_{s=1}^{r-2} \widetilde{A}_{2^{s}}=(N / 4)(3 r-5)-r+2 .
\end{aligned}
$$

Consequently, the $\operatorname{DFT}(N)$ of a real-valued sequence computed by our method requires

$$
\begin{gathered}
M_{N}^{r}=M_{N}^{c}+M_{N}^{s}=(N / 2)(r-3)+2, \\
A_{N}^{r}=A_{N}^{c}+A_{N}^{s}=(N / 2)(3 r-5)+4
\end{gathered}
$$


real multiplications and real additions, respectively. Further, by

$$
\begin{aligned}
& \hat{x}_{k}= \sum_{j=0}^{N-1} \operatorname{Re}\left(x_{j}\right) \cos (2 \pi k j / N)-\sum_{\substack{j=1 \\
j \neq N / 2}}^{N-1} \operatorname{Im}\left(x_{j}\right) \sin (2 \pi k j / N) \\
&+i\left(\sum_{j=0}^{N-1} \operatorname{Im}\left(x_{j}\right) \cos (2 \pi k j / N)+\sum_{\substack{j=1 \\
j \neq N / 2}}^{N-1} \operatorname{Re}\left(x_{j}\right) \sin (2 \pi k j / N)\right) \\
&(k=0, \ldots, N-1),
\end{aligned}
$$

the number of real operations of the $\operatorname{DFT}(N)$ of a complex-valued sequence is given by

$$
M_{N}=2 M_{N}^{r}=N(r-3)+4, \quad A_{N}=2 A_{N}^{r}+2(N-2)=3 N(r-1)+4 .
$$

Compared with other DFT-algorithms, we conclude that our polynomial algorithm works with the same computational complexity as the algorithm in [15] and the split-radix algorithm $[3,14]$.

\section{FAST ALGORITHM FOR THE DCT $(3 N)$}

The polynomial representations of the $\operatorname{DCT}(N)$, the cos-DFT $(N)$, and the $\sin -\operatorname{DFT}(N)$ in $\S \S 3$ and 4 open new possibilities for the derivation of fast algorithms for these transforms for various lengths $N$ by applying the CRT in combination with the factorizations (2.5), (2.6), and (2.7) of Chebyshev polynomials, or in combination with the factorization (2.11). The reductions of polynomials of the form (3.2) or (4.8) modulo Chebyshev polynomials in such algorithms can be performed only by (2.8), (2.9), and (2.10). In order to illustrate these general considerations, we suggest a new polynomial algorithm for the $\operatorname{DCT}(3 N)$.

We consider the $\operatorname{DCT}(3 N) \quad(N \in \mathbb{N})$, i.e., for given

$$
X:=\sum_{j=0}^{3 N-1} x_{j} T_{j} \bmod T_{3 N},
$$

we have to evaluate

$$
\tilde{x}_{k}=X(z) \bmod (z-\cos (\pi(2 k+1) / 6 N)) \quad(k=0, \ldots, 3 N-1) .
$$

By (2.5), $T_{3 N}$ factors as

$$
T_{3 N}=4\left(T_{N}-\sqrt{3} / 2\right) T_{N}\left(T_{N}+\sqrt{3} / 2\right),
$$

so that $X \bmod T_{3 N}$ is completely determined by the residues $X \bmod T_{N}$ and $X \bmod \left(T_{N} \pm \sqrt{3} / 2\right)$. Considering that by (2.5) and (2.8)

$$
\begin{aligned}
T_{2 N} & =2 T_{N}^{2}-1, \\
T_{N+j} & =2 T_{N} T_{j}-T_{N-j}, \\
T_{2 N+j} & =\left(4 T_{N}^{2}-1\right) T_{j}-2 T_{N} T_{N-j} \quad(j=1, \ldots, N-1),
\end{aligned}
$$

we obtain the following recursive algorithm. 
First, we calculate $X \bmod T_{N}$ by

$$
X^{(0)}:=\sum_{j=0}^{N-1} x_{j}^{(0)} T_{j}=X \bmod T_{N}
$$

with

$$
x_{j}^{(0)}:= \begin{cases}x_{0}-x_{2 N} & \text { for } j=0, \\ x_{j}-x_{2 N-j}-x_{2 N+j} & \text { for } j=1, \ldots, N-1 .\end{cases}
$$

Since by $(2.3), T_{N}$ has the roots $\cos (\pi(2 k+1) / 2 N)=c \quad s(\pi(2(3 k+1)+1) / 6 N)$ $(k=0, \ldots, N-1)$, we obtain

$$
\tilde{x}_{3 k+1}=X^{(0)} \bmod \left(T_{1}-\cos (\pi(2 k+1) / 2 N)\right) \quad(k=0, \ldots, N-1) .
$$

Next, we form $X \bmod \left(T_{N} \pm \sqrt{3} / 2\right)$ as follows:

$$
X^{(1)}:=\sum_{j=0}^{N-1} x_{j}^{(1)} T_{j}=X \bmod \left(T_{N}-\sqrt{3} / 2\right)
$$

with

$$
x_{j}^{(1)}:= \begin{cases}x_{0}+x_{2 N} / 2+x_{N} \sqrt{3} / 2 & \text { for } j=0, \\ x_{j}-x_{2 N-j}+2 x_{2 N+j}+\sqrt{3}\left(x_{N+j}-x_{3 N-j}\right) & \text { for } j=1, \ldots, N-1,\end{cases}
$$

and

$$
X^{(2)}:=\sum_{j=0}^{N-1} x_{j}^{(2)} T_{j}=X \bmod \left(T_{N}+\sqrt{3} / 2\right)
$$

with

$$
x_{j}^{(2)}:= \begin{cases}x_{0}+x_{2 N} / 2-x_{N} \sqrt{3} / 2, & \text { for } j=0, \\ x_{j}-x_{2 N-j}+2 x_{2 N+j}-\sqrt{3}\left(x_{N+j}-x_{3 N-j}\right) & \text { for } j=1, \ldots, N-1 .\end{cases}
$$

By (2.6), the zeros of $T_{N}-\sqrt{3} / 2$ and of $T_{N}+\sqrt{3} / 2$ are given by

$$
\begin{aligned}
& \{\cos ((\pi / 6+2 \pi k) / N): k=0, \ldots, N-1\} \\
& \quad=\left\{\cos (\pi(2(6 k)+1) / 6 N), \cos \left(\pi\left(2\left(6 k^{\prime}+5\right)+1\right) / 6 N\right):\right. \\
& \left.\quad k=0, \ldots, M ; k^{\prime}=0, \ldots, M^{\prime}\right\}
\end{aligned}
$$

and by

$$
\begin{aligned}
& \{\cos (5 \pi / 6+2 \pi k / N): k=0, \ldots, N-1\} \\
& \quad=\left\{\cos (\pi(2(6 k+2)+1) / 6 N), \cos \left(\pi\left(2\left(6 k^{\prime}+3\right)+1\right) / 6 N\right):\right. \\
& \left.\quad k=0, \ldots, M ; k^{\prime}=0, \ldots, M^{\prime}\right\},
\end{aligned}
$$


respectively, where $M:=\lceil N / 2\rceil-1$ and $M^{\prime}:=\lfloor N / 2\rfloor-1$. Hence, we get by (6.2) and (6.3) that

$$
\begin{aligned}
\tilde{x}_{6 k} & =X^{(1)} \bmod \left(T_{1}-\cos (\pi(12 k+1) / 6 N)\right) & & (k=0, \ldots, M), \\
\tilde{x}_{6 k+5} & =X^{(1)} \bmod \left(T_{1}-\cos (\pi(12 k+11) / 6 N)\right) & & \left(k=0, \ldots, M^{\prime}\right), \\
\tilde{x}_{6 k+2} & =X^{(2)} \bmod \left(T_{1}-\cos (\pi(12 k+5) / 6 N)\right) & & (k=0, \ldots, M), \\
\tilde{x}_{6 k+3} & =X^{(2)} \bmod \left(T_{1}-\cos (\pi(12 k+7) / 6 N)\right) & & \left(k=0, \ldots, M^{\prime}\right) .
\end{aligned}
$$

As a result, we have decomposed the $\operatorname{DCT}(3 N)$ into the $\operatorname{DCT}(N)$ given by (6.1) and into the modified $\operatorname{DCT}(N)$ 's determined by (6.2) and (6.3), with a total of $2 N$ multiplications and $6 N-2$ additions. Obviously, using (2.6) instead of (2.5), these modified DCT's can be handled similarly as the usual DCT. We have only to change the transform factors in the multiplications.

We now combine this idea with the developments of the previous sections. Let $N=2^{r} \quad(r \geq 2)$. Then by (3.7) and (3.8), we can perform the $\operatorname{DCT}(3 N)$ with

$\widetilde{M}_{3 N}=3 \widetilde{M}_{N}+2 N=(N / 2)(3 r+4), \quad \widetilde{A}_{3 N}=3 \widetilde{A}_{n}+6 N-2=(N / 2)(9 r+6)+1$

real operations. Using the decompositions in $\S 5$ (see Figure 4), we obtain the following computational complexity for the $\cos -\operatorname{DFT}(3 N)$ and for the sin$\operatorname{DFT}(3 N)$ :

$$
\begin{aligned}
& M_{3 N}^{c}=M_{3 N}^{s}=\sum_{s=0}^{r-2} \widetilde{M}_{3 \cdot 2^{s}}+2=(N / 4)(3 r-5)+3, \\
& A_{3 N}^{c}=3 N / 2-1+2 \sum_{s=0}^{r-2} 3 \cdot 2^{s}+\sum_{s=0}^{r-2} \tilde{A}_{3 \cdot 2^{s}}+8=(N / 4)(9 r-3)+r+6, \\
& A_{3 N}^{s}=3 N / 2-1+2 \sum_{s=0}^{r-2}\left(3 \cdot 2^{s}-1\right)+\sum_{s=0}^{r-2} \tilde{A}_{3 \cdot 2^{s}}+2=(N / 4)(9 r-3)-r+2 .
\end{aligned}
$$

Finally, we see that the $\operatorname{DFT}(3 N)$ requires

$$
M_{3 N}^{r}=(N / 2)(3 r-5)+6, \quad M_{3 N}=N(3 r-5)+12
$$

real multiplications and

$$
A_{3 N}^{r}=(N / 2)(9 r-3)+8, \quad A_{3 N}=N(9 r+3)+12
$$

real additions. This coincides with the number of real operations for the computation of the $\operatorname{DFT}\left(3 \cdot 2^{r}\right) \quad(r \geq 2)$ by combining the prime factor algorithm, the split-radix algorithm and the Rader algorithm [1, 14].

\section{BIBLIOGRAPHY}

1. A. V. Aho, J. E. Hopcroft, and J. D. Ullmann, The design and analysis of computer algorithms, Addison-Wesley, Reading, Mass., 1976.

2. G. Bruun, z-transform DFT filters and FFTs, IEEE Trans. Acoust. Speech Signal Process. 26 (1978), 56-63. 
3. P. Duhamel, Implementation of "split-radix" FFT algorithms for complex, real and realsymmetric data, IEEE Trans. Acoust. Speech Signal Process. 34 (1986), 285-295.

4. D. Garbe, On the level of irreducible polynomials over Galois fields, J. Korean Math. Soc. 22 (1985), 117-124.

5. I. S. Gradshtein and I. M. Ryzhik, Tables of integrals, sums, series and products, Nauka, Moscow, 1971. (Russian)

6. M. T. Heideman and C. S. Burrus, On the number of multiplications necessary to compute a length- $2^{n}$ DFT, IEEE Trans. Acoust. Speech Signal Process. 34 (1986), 91-95.

7. H. S. Hou, A fast recursive algorithm for computing the discrete cosine transform, IEEE Trans. Acoust. Speech Signal Process. 35 (1987), 1455-1462.

8. B. G. Lee, A new algorithm to compute the discrete cosine transform, IEEE Trans. Acoust. Speech Signal Process. 32 (1984), 1243-1245.

9. M. J. Narasimha and A. M. Peterson, On computing the discrete cosine transform, IEEE Trans. Comm. 26 (1978), 934-936.

10. H. J. Nussbaumer, Fast Fourier transform and convolution algorithms, Springer, BerlinHeidelberg-New York, 1981.

11. S. Paszkowski, Numerical application of Chebyshev polynomials and series, Nauka, Moscow, 1983. (Russian)

12. T. J. Rivlin, The Chebyshev polynomials, Wiley, New York-London-Sydney-Toronto, 1974.

13. H. V. Sorensen, D. L. Jones, C. S. Burrus, and M. T. Heideman, On computing the discrete Hartley transform, IEEE Trans. Acoust. Speech Signal Process. 33 (1985), 1231-1238.

14. H. V. Sorensen, D. J. Jones, M. T. Heideman, and C. S. Burrus, Real-valued fast Fourier transform algorithms, IEEE Trans. Acoust. Speech Signal Process. 35 (1987), 849-863.

15. M. Vetterli and H. J. Nussbaumer, Simple FFT and DCT algorithms with reduced number of operations, Signal Process. 6 (1984), 267-278.

16. S. Winograd, On computing the discrete Fourier transform, Math. Comp. 32 (1978), 175199.

17. __ Arithmetic complexity of computations, CBMS Regional Conf. Ser. in Math., vol. 33, SIAM, Philadelphia, 1980.

Fachbereich Mathematik, Universität Rostock, Universitätsplatz 1, 0-2500 Rostock, GeRMANY 